\title{
CONTAMINAÇÃO DE ÁGUAS SUPERFICIAIS POR AGROTÓXICOS: ANÁLISE DOS IMPACTOS CAUSADOS NA SAÚDE HUMANA E AMBIENTAL
}

\author{
Jacinta de Fátima Freitas Menezes ${ }^{(1)}$, João Victor Pena dos Santos ${ }^{\left({ }^{*}\right)}$, José Arthur de \\ Souza Santos Dutra ${ }^{(I)}$, Matheus Gomes Tavares ${ }^{(I)}$ \& Heloisa Alves Guimarães ${ }^{(l)}$
}

\section{RESUMO}

MENEZES, J.F.F; DOS SANTOS, J.V.P.; DUTRA, J.A.S.S.; TAVARES, G.T.; GUIMARÃES, H.A. Contaminação de águas superficiais por agrotóxicos: análise dos impactos causados na saúde humana e ambiental. Perspectivas Online: Biológicas \& Saúde, v.11 , n.37, p.19-35, 2021.

Atualmente o Brasil é um dos maiores consumidores de agrotóxicos do mundo, pesquisadores vêm alertando sobre os problemas causados pelo uso intensivo destas substâncias, que tem fomentado uma grande preocupação em diversos setores devido aos riscos potenciais que estes compostos trazem ao ambiente e aos seres humanos, uma vez que boa parte desses agrotóxicos vem sendo associados a doenças crônicas como câncer, defeitos congênitos e distúrbios endócrinos. Apesar disso, cada vez mais estes compostos são utilizados para o controle da integridade de plantações. Estudos comprovam um aumento constante de águas contaminadas por agrotóxicos, que por meio da lixiviação e do escoamento superficial são transportados, prejudicando a qualidade das águas subterrâneas e superficiais. É o caso do município de Campos dos Goytacazes,
Rio de Janeiro (RJ), Brasil, onde de acordo com estudo nacional realizado para determinar a presença de agrotóxicos na água, é uma das cidades nas quais 27 tipos de agrotóxicos chegam à suas torneiras para consumo humano, e em determinados casos, os agrotóxicos encontram-se acima dos limites legais existentes no Brasil. Diante deste cenário, este artigo tem como objetivo apresentar e discutir os efeitos da exposição aos diversos agrotóxicos encontrados nas águas superficiais do município. As discussões e apontamentos do trabalho poderão contribuir para demonstrar a grande degradação da qualidade de recursos hídricos da região, causada pelo uso intensivo de agrotóxicos, incluindo fontes de água de consumo humano e de chuvas, amplificando o risco de contaminação.

Palavras-chave: Doenças crônicas; Campos dos Goytacazes; Agricultura; Recursos hídricos.

${ }^{1}$ Instituto Federal Fluminense Campus Guarus- IFF/Guarus - Av. Souza Mota, 350 - Parque Fundão - Campos dos GoytacazesRJ, Brasil, CEP: 28060-010 (*) e-mail: jvpenasantos@gmail.com

Data de recebimento: 08/12/2021. Aceito para publicação: 21/06/2021. Data de publicação: 30/06/2021.

Persp. Online: biol. \& saúde, Campos dos Goytacazes, 37 (11) 19-35, 2021

seer.perspectivasonline.com.br 


\title{
CONTAMINATION OF SURFACE WATER BY PESTICIDES: ANALYSIS OF IMPACTS CAUSED ON HUMAN AND ENVIRONMENTAL HEALTH
}

\author{
Jacinta de Fátima Freitas Menezes ${ }^{(1)}$, João Victor Pena dos Santos ${ }^{\left(1^{*}\right)}$, José Arthur de \\ Souza Santos Dutra ${ }^{(1)}$, Matheus Gomes Tavares ${ }^{(1)}$ \& Heloisa Alves Guimarães ${ }^{(1)}$
}

\begin{abstract}
MENEZES, J.F.F; DOS SANTOS, J.V.P.; DUTRA, J.A.S.S.; TAVARES, G.T.; GUIMARÃES, H.A. Contaminação de águas superficiais por agrotóxicos: análise dos impactos causados na saúde humana e ambiental. Perspectivas Online: Biológicas \& Saúde, v.11 , n.37, p.19-35, 2021.

Currently Brazil is one of the largest consumers of pesticides in the world, researchers have been warning about the problems caused by the intensive use of these substances, which has fostered a great concern in several sectors due to the potential risks that these compounds bring to the environment and to humans, since much of these pesticides have been associated with chronic diseases such as cancer, congenital defects and endocrine disorders. However, more and more are used to control the integrity of plantations. The studies have already demonstrated a surface change in water contaminated by pesticides, which can be transported through leaching and surface runoff, damaging seed and surface quality. This is

Goytacazes, RJ, where the national work is carried out to determine the presence of pesticides in the water, is one of the cities which 27 agrochemicals that arrives in their taps for human consumption, and in certain cases, pesticides are above the legal limits existing in Brazil. In view of this scenario, this article aims to present and discuss the effects of exposure to the various pesticides found in the surface waters of the municipality. The discussions and notes of the work may contribute to demonstrate the great degradation of the quality of water resources in the region, caused by the intensive use of pesticides, including sources of water for human consumption and rainfall, amplifying the risk of contamination.
\end{abstract} the case of the municipality of Campos dos

Keywords: Chronic diseases; Campos dos Goytacazes; Agriculture; Water resources.

${ }^{1}$ Fluminense Federal Institute, Guarus College, FFI/Guarus - Av. Souza Mota, 350 - Parque Fundão - Campos dos GoytacazesRJ, Brazil, Postal Code: 28060-010 (*) e-mail: jvpenasantos@gmail.com

Received: 12/8/2021.

Publication in: 30/06/2021. 


\section{INTRODUÇÃO}

Por meio da necessidade de desenvolvimento da sociedade humana, o principal foco de investimento e aperfeiçoamento se voltou para o setor da agricultura para suprir as necessidades da população. Por meio da Revolução Industrial (RI), viu-se a urgência no foco do desenvolvimento da agricultura buscando aperfeiçoar a produção alimentar em virtude do crescimento populacional (CHAIM, 1999).

No passado os métodos utilizados na produção agrícola com o objetivo de evitar a proliferação de pragas eram baseados na aplicação de produtos químicos à base de Cobre e Arsênio, assim como a aplicação das inseticidas de origem natural como as piretrinas (SILVA; SANTOS, 2007).

Com o surgimento de novas tecnologias e a evolução do próprio homem como produtor, diversas técnicas foram sendo desenvolvidas, com o objetivo de facilitar e seguir de forma paralela a relação produção de alimentos-crescimento demográfico, a fim de garantir a subsistência das gerações presentes e futuras (PEDLOWSKI et al., 2006). Isso resultou em uma grande transformação tecnológica no setor dos sistemas produtivos, que começaram a contar com a utilização dos agrotóxicos, que se tornaram essenciais na redução do tempo de trabalho e no combate de pragas na produção.

Um dos principais produtores e consumidores desses agrotóxicos são os países desenvolvidos, que com o objetivo de suprir a necessidade da sua população e evitar as perdas por insetos e pragas, os quais geram uma destruição de cerca de $20 \%$ das lavouras, buscam assim garantir o fornecimento adequado do alimento para as pessoas (OLIVEIRA; MACEDO, 2011). O uso desenfreado de agrotóxicos leva a diversos impactos socioambientais que, apesar de graves, não impedem o uso e a disseminação dessas substâncias em todo o mundo (LOPES; ALBUQUERQUE, 2018).

$\mathrm{Na}$ época da RI o principal meio de produção era através de monoculturas, que deu o ponta pé inicial na agricultura. Com o passar dos anos uma nova substância permitiu a produção em massa e as perdas quase inexistentes, atreladas à produção em grande escala. Conhecidas como agrotóxicos, estas substâncias supriram a demanda exacerbada de alimentos em todo o mundo, permitindo uma produção em larga escala na agricultura e impedindo a proliferação de pragas durante o processo (ECOBICHÓN, 2001).

Porém o custo socioambiental causado pela alta toxicidade de tais produtos, unida à falta de informações sobre possíveis riscos envolvidos na sua utilização devido a carência acerca da maneira correta de aplicá-los, é um fato que predomina entre os agricultores e que tem sido responsável pelo elevado grau de contaminação ao meio ambiente e à saúde humana, sendo o ser humano uma das principais vítimas afetadas pelo seu uso constante e irresponsável (WORLD RESOURCES INSTITUTE, 1998).

Essa contaminação se dá em três tipos de graus de intoxicação definidas pelo Manual de Vigilância da Saúde de Populações Expostas a Agrotóxicos, produzido pela Organização Pan-Americana de Saúde (OPAS) como: aguda, subaguda e crônica, que vai depender do tempo

Persp. Online: biol. \& saúde, Campos dos Goytacazes, 37 (11) 19-35, 2021

seer.perspectivasonline.com.br 
de exposição e a toxicidade das substâncias manuseadas (ORGANIZAÇÃO PANAMERICANA DA SAÚDE, 1996).

Tais intoxicações estão relacionadas a problemas como surgimento de cânceres, danos ao sistema nervoso e desenvolvimento de fetos e bebês com malformações, por exemplo. Estes são alguns dos muitos problemas decorrentes da contaminação por agrotóxicos, com o agravante que algumas doenças e sintomas, ou não podem ser diagnosticados ou não se manifestam na pessoa contaminada, podendo mostrar sintomas de contaminação entre 15 a 30 anos após o contato do produto com o corpo. Além disso, o estabelecimento de doses fatais para seres humanos é seguido de uma identificação dos níveis de toxicidade de cada produto (PEDLOWSKI et al., 2006).

O Brasil, um dos maiores consumidores de agrotóxicos, representa sozinho $50 \%$ do consumo na região da América do Sul. Após a "revolução verde" tornou-se um grande produtor e contaminador socioambiental, pelo seu extensivo uso de produtos agrotóxicos nos seus processos agrícolas (PERES; MOREIRA, 2003). No entanto, a destreza técnica para manusear os produtos químicos e os processos não tiveram qualificação necessária, engatando uma série de problemas ambientais e sociais imediatos na população brasileira (PERES et al., 2001).

Os agrotóxicos no Brasil começaram a ser inseridos na agricultura em 1943, quando o DDT (Diclorodifeniltricloroetano) foi introduzido no país, e atualmente possui uma faixa de consumo de aproximadamente 300 mil toneladas de agrotóxicos por ano (SPADOTTO, 2006).

O DDT possui a fórmula molecular $\left(\mathrm{C}_{14} \mathrm{H}_{9} \mathrm{Cl}_{5}\right)$ sendo estruturalmente composto por dois anéis aromáticos que se encontram ligados a um átomo de cloro cada, e um átomo de carbono ligado a outros 3 átomos de cloro, como pode ser observado na (Figura 1) (TEIXEIRA et al., 2019).

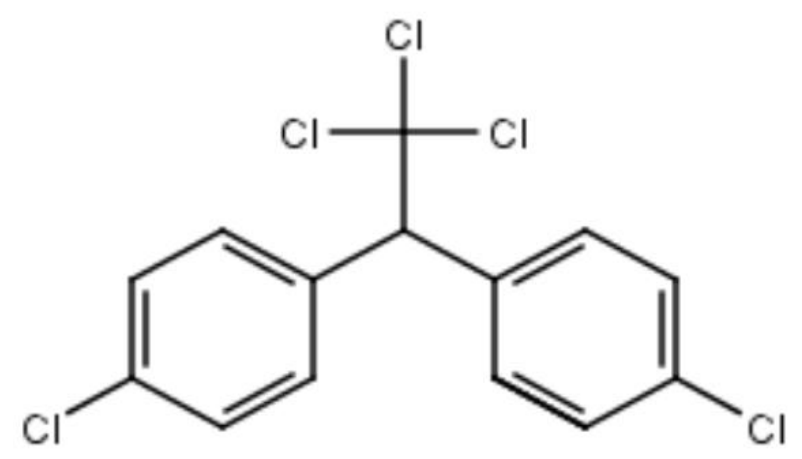

Figura 1: Fórmula estrutural do Diclorodifeniltricloroetano (DDT).

(Fonte: TEIXEIRA et al.,2019).

Devido ao seu baixo custo e sua alta eficiência, o DDT foi introduzido no Brasil na década de 40 no combate às pragas agrícolas nas diversas culturas como eficiente inseticida, sendo incorporado pelo Ministério da Agricultura entre os principais agrotóxicos comercializados (MORAES, 2019). No entanto, a partir de 1971 os efeitos da contaminação pelo DDT chegaram a níveis exorbitantes devido às consequências causadas em insetos e aves,

Persp. Online: biol. \& saúde, Campos dos Goytacazes, 37 (11) 19-35, 2021

seer.perspectivasonline.com.br 
e aos vários distúrbios provocados na população humana, tais como, alterações de comportamento, distúrbios sensoriais, da alteração da atividade da musculatura involuntária, depressão dos centros vitais e problemas no sistema nervoso central. Diversos outros sintomas podem se manifestar de acordo com o tempo de exposição ao DDT, ao método de absorção do produto no corpo e pela via de entrada no organismo (AMATO et al., 2002).

A dispersão ambiental do DDT pode ocorrer por meio atmosférico, oceânico e nos diversos tipos de solos, além de ser transportada através da bioacumulação em diversos tipos de animais (AMATO et al., 2002).

Os efeitos da contaminação por agrotóxicos à saúde acarretam uma série de consequências, podendo ser agudos ou crônicos. Os agudos são provenientes de exposição que causam os efeitos visíveis em menos de 24 horas, como convulsões e espasmos musculares, por exemplo, (BRASIL, 1996). Os efeitos crônicos são aqueles provenientes de exposição com pequenas doses contínuas de produtos, apresentando resultados da exposição após semanas, meses ou até anos, sendo mais difíceis de serem diagnosticados.

Os agrotóxicos são geralmente classificados por meio do tipo de praga que controlam, podendo ser: inseticidas, fungicidas e herbicidas, com cada qual contendo diferentes moléculas orgânicas ou inorgânicas, que geram distintos tipos de danos à saúde humana (PERES; MOREIRA, 2003).

Langenbach (1999) indica que as estatísticas têm mostrado que o número de pragas nas lavouras está aumentando, apesar do uso de agrotóxicos, isso porque as mesmas estão desenvolvendo mecanismos de resistência aos produtos.

No momento presente, o Brasil ainda possui políticas públicas que fomentam o uso e o comércio de agrotóxicos. Na última década, o Brasil expandiu em 190\% o mercado de agrotóxicos, o que colocou o país em primeiro lugar no ranking mundial de consumo desde 2008. Dez empresas controlam mais de $70 \%$ desse mercado no país. Somente na safra produzida nos anos de 2010 e 2011, foram consumidas 936 mil toneladas de agrotóxicos (RIGOTTO et al., 2014).

No Brasil, observa-se na região Sul um consumo de aproximadamente $30 \%$ de agrotóxicos com relação ao total consumido no país. Sendo o estado do Paraná, com relação aos demais, o maior consumidor, com um volume de 12 quilos por hectare/ano, diante de uma média brasileira de 4 quilos/hectare/ano (NEVES; BELLINI, 2013).

Os inseticidas da classe dos organofosforados e carbamatos são os principais responsáveis pela chamada crise colinérgica, decorrente da inibição das enzimas responsáveis pela degradação de acetilcolina, um neurotransmissor cerebral que atua de forma imprescindível para o funcionamento de estruturas como a memória e a função motora do corpo, o que ocasionam diversas alterações no sistema nervoso originando os sintomas previstos no (Quadro 1) (PERES; MOREIRA, 2003). 
Quadro 1: Efeitos da exposição aos agrotóxicos.

\begin{tabular}{|c|c|c|c|}
\hline $\begin{array}{c}\text { Classificação Quanto } \\
\text { À Praga Que } \\
\text { Controla }\end{array}$ & $\begin{array}{l}\text { Classificação Quanto Ao } \\
\text { Grupo Químico }\end{array}$ & $\begin{array}{c}\text { Sintomas De Intoxicação } \\
\text { Aguda }\end{array}$ & $\begin{array}{c}\text { Sintomas De Intoxicação } \\
\text { Crônica }\end{array}$ \\
\hline \multirow{8}{*}{ Inseticidas } & \multirow{5}{*}{$\begin{array}{l}\text { Organofosforados e } \\
\text { carbamatos }\end{array}$} & Fraqueza & $\begin{array}{l}\text { Efeitos neurotóxicos } \\
\text { retardados }\end{array}$ \\
\hline & & Cólicas abdominais & \multirow[t]{2}{*}{ Alterações cromossomiais } \\
\hline & & Vômitos & \\
\hline & & Espasmos musculares & \multirow[t]{2}{*}{ Dermatites de contato } \\
\hline & & Convulsões & \\
\hline & \multirow{4}{*}{ Organoclorados } & Náuseas & Lesões hepáticas \\
\hline & & Vômitos & Arritmias cardíacas \\
\hline & & \multirow{2}{*}{$\begin{array}{l}\text { Contrações musculares } \\
\text { involuntárias }\end{array}$} & Neuropatias periféricas \\
\hline \multirow{10}{*}{ Fungicidas } & & & Lesões renais \\
\hline & \multirow{3}{*}{ Piretróides sintético } & Irritações das conjuntivas & Alergias \\
\hline & & Espirros & Asma brônquica \\
\hline & & Excitação & Irritações nas mucosas \\
\hline & \multirow{5}{*}{ Ditiocarbamatos } & Convulsões & Hipersensibilidade \\
\hline & & Tonteiras & Alergias respiratórias \\
\hline & & Vômitos & Dermatites \\
\hline & & Tremores musculares & Doença de Parkinson \\
\hline & & Dor de cabeça & Cânceres \\
\hline & Fentalamidas & & Teratogêneses \\
\hline \multirow[t]{11}{*}{ Herbicidas } & \multirow{3}{*}{$\begin{array}{l}\text { Dinitrofenóis e } \\
\text { pentaclorofenol }\end{array}$} & Dificuldade respiratória & \multirow{2}{*}{$\begin{array}{c}\text { Cânceres (PCP - formação } \\
\text { de dioxinas) }\end{array}$} \\
\hline & & Hipertermia & \\
\hline & & Convulsões & Cloroacnes \\
\hline & & Perda do apetite & Indução da produção de \\
\hline & Fenoxiacéticos & Enjôo & enzimas hepáticas \\
\hline & Fenoxiaceticos & Vômitos & Cânceres \\
\hline & & Fasciculação muscular & Teratogênese \\
\hline & & Sangramento nasal & Les̃̃es henáticas \\
\hline & Diniridilos & Fraqueza & Lesoes mepautas \\
\hline & Dipiriailos & Desmaios & Dermatites de contato \\
\hline & & Conjuntivites & Fibrose pulmonar \\
\hline
\end{tabular}

Fonte: WORLD HEALTH ORGANIZATION (WHO) (1990).

Porém a classe de inseticidas organoclorados possui efeitos mais crônicos, sendo acumulados no organismo humano, apresentando efeitos a longo prazo, em média de trinta anos (BRASIL, 1996). Nesta classe, há o acúmulo da toxina no tecido adiposo de forma 
bioacumulativa, aumentando a cadeia de contaminação nos animais e seres humanos. Por meio disso, o uso desse tipo de agrotóxico se tornou restrito e até proibido em muitos países (PERES; MOREIRA, 2003).

Esses efeitos à saúde humana são intensificados quando a população receptora prioritária que entra em contato com os produtos tóxicos não possui uma infraestrutura ou condições socioeconômicas adequadas à saúde, e acabam sofrendo as consequências da aplicação dos agrotóxicos na agricultura (VEIGA et al., 2006).

A aplicação dos agrotóxicos no solo pode dar-se por meio de três vias: sólida, líquida e gasosa, da qual o método mais comum utilizado é a aplicação por via líquida, sendo que a aplicação por via gasosa somente é utilizada em certos casos. O meio de aplicação por via líquida se dá por uma formulação que vai ser diluída em água, formando a chamada "calda", que em formas de gotas é aplicada ao solo por meio da pulverização (SPADOTTO, 2006).

Após a aplicação dos produtos no meio ambiente, sua disseminação vai depender dos processos de atraso ou impedimento da chegada do produto ao alvo, como desvio de rota, erro de rota, dentre outros. Na busca por uma melhor aplicação visando a redução do impacto ambiental e a perda do agrotóxico no meio ambiente, deve-se analisar a questão da aplicabilidade a fim de evitar essas perdas (GEBLER; SPADOTTO, 2004).

A contaminação do meio ambiente pelo uso constante e irresponsável de agrotóxicos ocorre em muitos casos pela mistura irresponsável dos produtos em empresas ou lavouras, o que pode resultar em problemas a longo prazo ou até mesmo danos irreversíveis (NOGUEIRA; BRANCO, 2011). Essas consequências prejudicam tanto os seres bióticos quanto os seres abióticos dentro dos ecossistemas do planeta. Gerando com isso, uma influência negativa com as populações existentes no meio ambiente (PERES; MOREIRA, 2003).

Uma das contaminações mais significativas e que geram uma proliferação secundária na disseminação dos agrotóxicos e dos sintomas ligados a ele é a que ocorre na água. A Portaria do Ministério da Saúde $n^{\circ}$ 518/2004 regulamenta a respeito da potabilidade da água: ela estabelece as responsabilidades por parte de quem produz a água, a quem cabe o exercício do controle de qualidade da água e das autoridades sanitárias, com a missão de "vigilância da qualidade da água" para consumo humano (NETO; SARCINELLI, 2009).

A água é um recurso que sempre entra em contato com diversos tipos de contaminantes, de origem tanto orgânica quanto inorgânica provenientes de diversos processos que ocorrem no ambiente e são lixiviados ou depositados no corpo hídrico. Os compostos de origem inorgânica enquadram os contaminantes gerados na agricultura tais como, nitratos, fosfatos, metais pesados de origem industrial, mineração, produtos farmacêuticos, fertilizantes, provenientes de atividades de termelétricas, dentre outros. Já os compostos orgânicos são os provenientes de lodos de estações de tratamento de efluentes (ETE), atividades petroquímicas, agrotóxicos, entre outros (STEFFEN et al., 2011).

A contaminação da água pelo uso de agrotóxicos se inicia pela contaminação do solo, e posteriormente, através da infiltração, lixiviação, bioacumulação, biomagnificação e difusão, esses contaminantes chegam aos corpos hídricos (STEFFEN et al., 2011).

Persp. Online: biol. \& saúde, Campos dos Goytacazes, 37 (11) 19-35, 2021

seer.perspectivasonline.com.br 
Além disso, estes podem ser transportados tanto por correntes d'água quanto presos ao corpo de organismos aquáticos, e por meio da volatização podem retornar para a atmosfera na forma de moléculas inorgânicas, reforçando assim a ideia da existência de uma interação entre água, sedimentos e agrotóxicos (SILVA et al., 2013).

O solo é o agente responsável por diversas reações físicas, químicas e biológicas, além de promover interações com os minerais e constituintes orgânicos. As características apresentadas pelos seus constituintes que possuem grande reatividade, propiciam que haja constantemente uma série de reações no mesmo. Porém alguns tipos de substâncias não biodegradáveis acabam sendo transportados para outros meios aumentando assim o raio de contaminação neste ambiente (STEFFEN et al., 2011).

Os agrotóxicos lixiviados e percolados que alcançam os cursos d'água são a porta de entrada para a contaminação das populações. E dependendo dos níveis de concentração desses poluentes tóxicos podem-se causar diversos sintomas como apresentados anteriormente. As águas superficiais de abastecimento populacional são um alvo direto à destinação destes produtos após os processos de transporte e deposição. Isso afeta a qualidade do curso hídrico e ocasiona danos à saúde, sendo necessário a análise e tratamento antes da distribuição da água para o abastecimento público (NASCIMENTO et al., 2015).

Um estudo realizado recentemente no Brasil, em busca da catalogação e análise dos agrotóxicos nos corpos hídricos que chegam nas casas dos cidadãos, causou um alarme sobre a quantidade de contaminantes na água e sobre a qualidade da mesma para o consumo da sociedade. Uma das cidades analisadas neste estudo foi o município de Campos dos Goytacazes.

Com isso, o objetivo principal deste trabalho é explicar a relação entre o uso indiscriminado dos agrotóxicos com o aumento na incidência de contaminação da água e sua relação com a saúde humana, com base em estudos realizados no município de Campos dos Goytacazes - RJ.

\section{METODOLOGIA}

\subsection{Tipo de Estudo:}

O trabalho desenvolvido seguiu os preceitos do estudo exploratório, por meio de uma pesquisa de revisão bibliográfica a partir de material já elaborado, constituído de livros e artigos científicos.

Nesta perspectiva, seguiu-se as seguintes etapas:

\section{$1^{a}$ Etapa: Fonte}

A seguir está descrito a fonte que forneceu as respostas adequadas à solução do problema proposto: 
Foram utilizados artigos científicos como base de pesquisa ao tema proposto e que levassem ao entendimento do que está acontecendo no município de Campos dos Goytacazes, acessados na base de dados Scielo, publicados nos últimos 29 anos (1990 a 2015).

Utilizaram-se artigos nacionais e internacionais, disponíveis online em texto completo. Para a seleção das fontes, foram consideradas como critério de inclusão as bibliografias que abordassem a contaminação de águas superficiais por meio de agrotóxicos, sendo descartadas aquelas que não atenderam a temática.

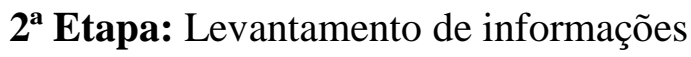

A seleção dos conteúdos se seguiu da seguinte forma:

a) Leitura exploratória (leitura rápida que objetiva verificar se a obra consultada se relaciona com o tema proposto);

b) Avaliação da qualidade dos conteúdos (análise mais aprofundada do material selecionado buscando as partes que realmente interessam);

c) Registro das informações extraídas das fontes em instrumento específico (autores, ano, método, resultados e conclusões).

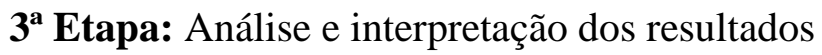

Nesta etapa foi realizada uma leitura analítica com finalidade de ordenar e sumariar as informações contidas nas fontes, de forma que estas possibilitaram a obtenção de respostas ao problema da pesquisa.

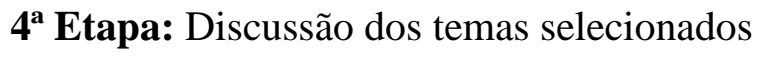

Categorias que emergiram da etapa anterior foram analisadas e discutidas a partir do referencial teórico relativo à temática do estudo.

\section{RESULTADOS E DISCUSSÃO}

No estudo realizado no Brasil para o levantamento dos agrotóxicos encontrados nos corpos hídricos responsáveis pelo abastecimento populacional, a cidade de Campos dos Goytacazes - RJ ganhou destaque, sendo considerada uma dentre as 19 cidades no Estado do Rio de Janeiro que apresentaram todas as substâncias analisadas no chamado "coquetel tóxico" contendo 27 agrotóxicos, presentes na água de consumo. 11 destes estão associados a doenças crônicas (Figura 2), os outros 16 estão representados na Figura 3. 


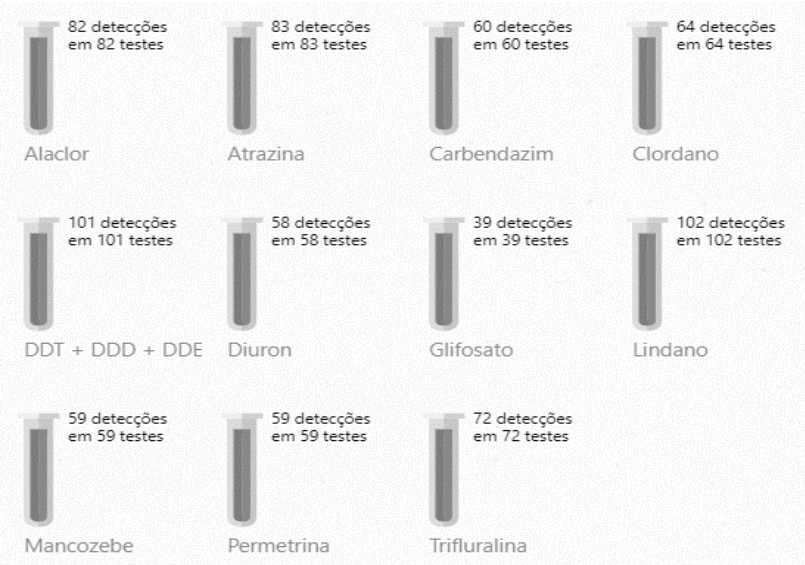

Figura 2: Agrotóxicos detectados no município de Campos dos Goytacazes, no estado do Rio de Janeiro, Brasil, associados a doenças crônicas.

(Fonte: ARANHA; ROCHA, 2019).

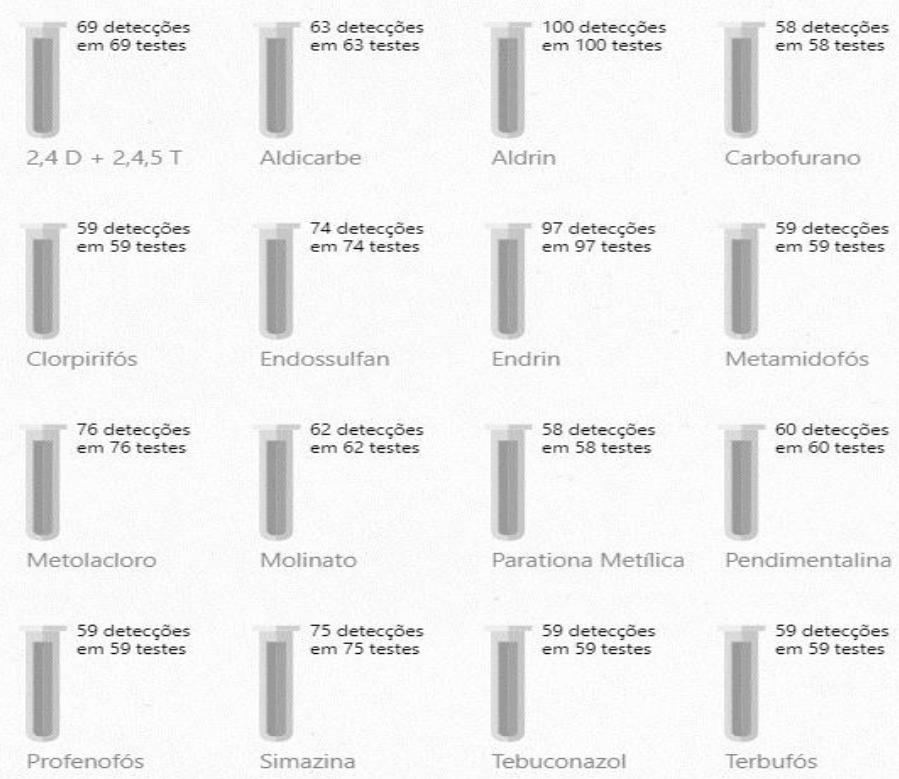

Figura 3: Outros 16 agrotóxicos detectados no município de Campos dos Goytacazes, no estado do Rio de Janeiro, Brasil.

(Fonte: ARANHA; ROCHA, 2019).

Deste total de 27 agrotóxicos, 9 estavam em concentrações superiores aos estabelecidos na legislação brasileira a respeito da potabilidade de água para consumo no período de 20142017 e 11 foram encontrados acima dos limites estabelecidos pela União Europeia (Figuras 4 e 5). Campos dos Goytacazes aparece como uma das 1.400 cidades que apresentaram os maiores níveis de contaminação da água distribuída para a população das mais de 2.700 cidades analisadas em todo o país (PEDLOWSKI et al., 2006). 

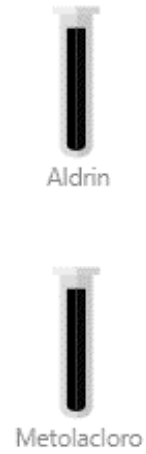
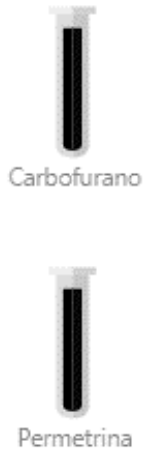
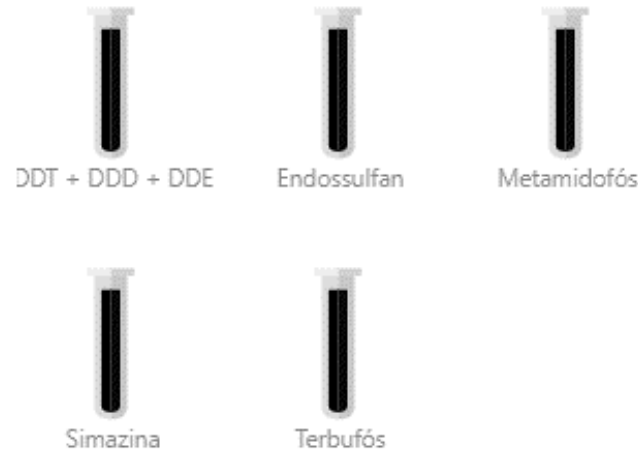

Figura 4: Agrotóxicos detectados em concentração superior ao limite considerado seguro no Brasil, em águas no município de Campos dos Goytacazes, estado do Rio de Janeiro, Brasil, entre 2014 e 2017.

(Fonte: ARANHA; ROCHA, 2019).

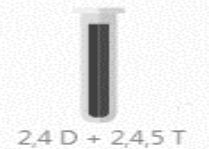

a.4.
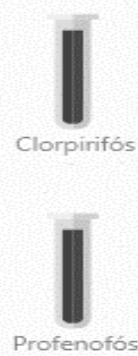
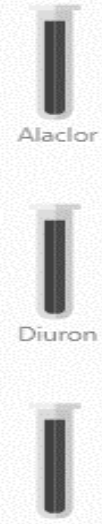

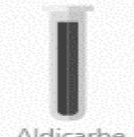

Aldicarbe
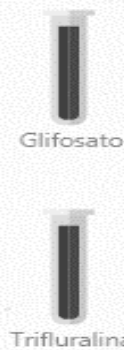
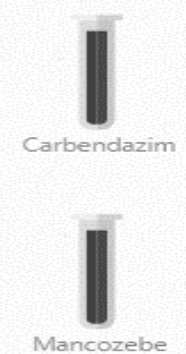

Figura 5: Agrotóxicos detectados em concentração superior ao limite considerado seguro na Europa em águas no município de Campos dos Goytacazes, estado do Rio de Janeiro, Brasil, entre 2014 e 2017.

(Fonte: ARANHA; ROCHA, 2019).

Dentre os 27 agrotóxicos identificados, 11 são responsáveis pela ocorrência de doenças crônicas como câncer, distúrbios endócrinos e malformação congênita. Pode-se perceber que o tamanho da exposição da população da cidade à contaminação deve-se, principalmente à forma indiscriminada que estes químicos chegam às torneiras das casas sem nenhum tratamento preventivo.

Na figura seguir, observa-se o sobressalto do uso indiscriminado dos agrotóxicos ao longo dos anos no Brasil. Em uma análise mais pontual se comparado o ano de 2014 ao de 2017 percebe-se um aumento de mais de $250 \%$ no número de agrotóxicos, onde nota-se que o maior nível de agrotóxicos em 14 anos.

Persp. Online: biol. \& saúde, Campos dos Goytacazes, 37 (11) 19-35, 2021

seer.perspectivasonline.com.br 


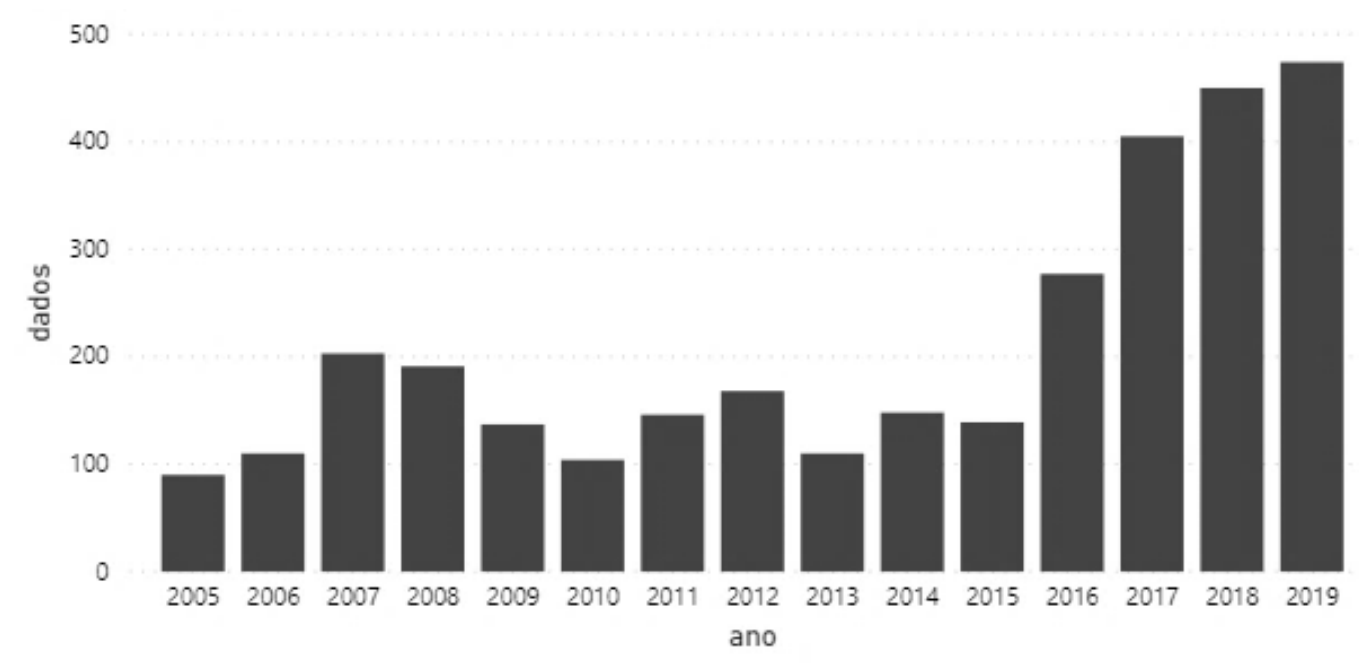

Figura 6: Total de agrotóxicos registrados no Brasil entre 2005 e 2019.

(Fonte: MOREIRA, 2019).

Esse aumento contínuo na produção e liberação da comercialização dos agrotóxicos, que nos últimos anos vê sua curva de formação voltada tanto para o ramo biológico como para o orgânico, tem como uma de suas justificativas uma tentativa de produção mais abrangente para os tipos de agricultura orgânica e tradicional (SUDRÉ, 2019).

O aumento progressivo no número de agrotóxicos produzidos e liberados no Brasil nos últimos anos vem do próprio incentivo do governo federal, que liberou no ano de 2019 mais de 57 novos tipos de agrotóxicos, dentre os quais 22 são proibidos na União Europeia (UE), e mais de 400 autorizações apenas durante o governo do presidente Bolsonaro para a liberação do uso de diversos tipos de químicos, número recorde desde 2005 (SUDRÉ, 2019).

A liberação associada a falta de conscientização dos produtores agrícolas que visam a primeiro prazo o lucro e a produtividade acabam por elevar a contaminação do solo e dos corpos hídricos que envolvem as plantações, vão disseminando aos poucos estes químicos para regiões mais longínquas e por fim se bioacumulando nos organismos vivos, desenvolvendo uma série de complicações a curtos e longo prazo.

Dentre os principais agrotóxicos proibidos na UE e liberados para uso e comercialização no Brasil um deles foi encontrado em Campos dos Goytacazes, a Atrazina, banida a mais de uma década da UE devido ao risco pela contaminação de águas subterrâneas e sua associação ao desenvolvimento de doenças crônicas. Apesar de não estar classificada acima do limite seguro estabelecida pela EU $(0.1 \mu \mathrm{g} / \mathrm{L})$ durante o levantamento da pesquisa realizada na cidade, o fato de estar presente na água destinada ao consumo humano já prova que há um risco à saúde.

No Quadro 2, observam-se os agrotóxicos liberados no Brasil em comparação a outros países onde seguem proibidos.

Quadro 2: Uso de agrotóxicos liberados no Brasil e proibidos em outros países.

BRASIL UE EUA CAN ARG AUS ÍND

Persp. Online: biol. \& saúde, Campos dos Goytacazes, 37 (11) 19-35, 2021

seer.perspectivasonline.com.br 


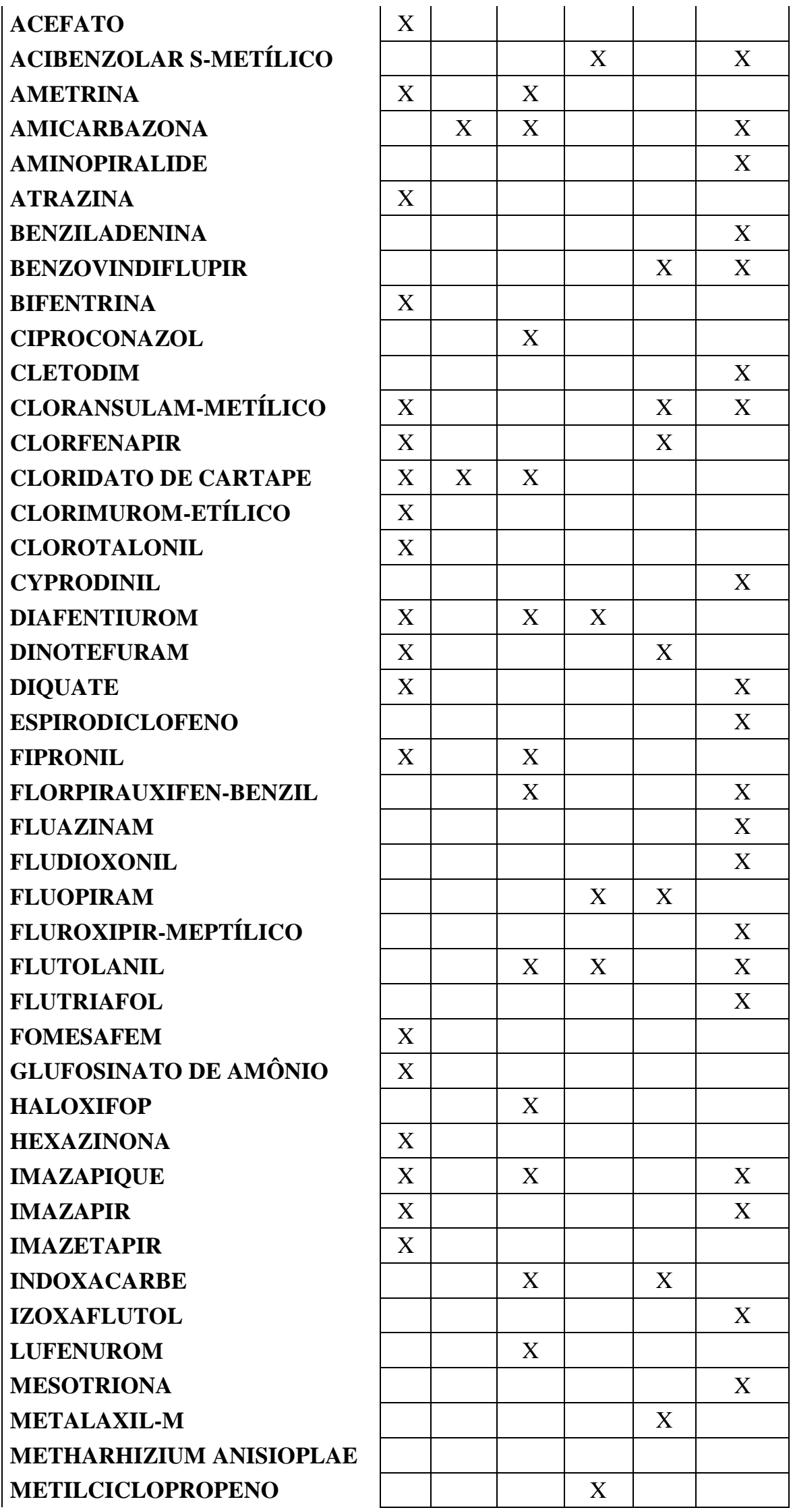




\begin{tabular}{|c|c|c|c|c|c|c|}
\hline \multirow{2}{*}{$\begin{array}{l}\text { METOMIL } \\
\text { METOXIFENOZINA }\end{array}$} & \multirow[t]{2}{*}{$\mathrm{X}$} & & & & & \multirow[t]{2}{*}{$\mathrm{X}$} \\
\hline & & & & & $X$ & \\
\hline NICOSSULFURON & & & & & $\mathrm{X}$ & $\mathrm{X}$ \\
\hline NOVALUROM & $\mathrm{X}$ & & & & & \\
\hline PECLOBUTRAZOL & & & & $\mathrm{X}$ & & \\
\hline PAECILOMYCES LILACINUS & & & $\mathrm{X}$ & $\mathrm{X}$ & $\mathrm{X}$ & $\mathrm{X}$ \\
\hline PICLORAM & & & & & & $\mathrm{X}$ \\
\hline PICOXISTROBINA & $\mathrm{X}$ & & & & & \\
\hline PROPANIL & $\mathrm{X}$ & & $\mathrm{X}$ & & & \\
\hline QUIZALOFOPE-P & & $\mathrm{X}$ & $\mathrm{X}$ & $\mathrm{X}$ & & $\mathrm{X}$ \\
\hline QUIZALOFOPE-P-TEFURÍLICO & & $\mathrm{X}$ & $\mathrm{X}$ & & X & \\
\hline SULFENTRAZONA & $\mathrm{X}$ & & & & & \\
\hline TEBUTIUROM & $\mathrm{X}$ & & X & & & $\mathrm{X}$ \\
\hline TIABENDAZOL & & & & & $\mathrm{X}$ & $\mathrm{X}$ \\
\hline TIAMETOXАM & $\mathrm{X}$ & & & & & \\
\hline TIODICARBE & $\mathrm{X}$ & & $\mathrm{X}$ & & & \\
\hline TRICHODERMA ASPERELLUM & & & & $\mathrm{X}$ & & \\
\hline TRICHODERMA HARZIANUM & & & & & $\mathrm{X}$ & \\
\hline TRICLOPIR & & & & & & $\mathrm{X}$ \\
\hline TRINEXAPAQUE-ETÍLICO & & & & & & $\mathrm{X}$ \\
\hline
\end{tabular}

EU = União Europeia; EUA = Estados Unidos da América do Norte $;$ CAN = Canadá; ARG = Argentina; AUS = Austrália; ÍND = Índia

Fonte: Adaptado de MOREIRA (2019).

Dentre os 63 agrotóxicos listados e liberados para uso no Brasil (Quadro 2), 27 não são permitidos na UE, 13 na Austrália, 29 na Índia e 18 no Canadá (MOREIRA, 2019). Dentre os permitidos no Brasil, muitos são tóxicos e causam problemas à saúde humana, além de desequilíbrios ambientais, como por exemplo, o Florpirauxifen-benzil e o Fluopiram, classificados pela Agência Nacional de Vigilância Sanitária (ANVISA) como substâncias altamente ou mediamente tóxicas para o homem e as diversas formas de organismos naturais nos diversos biomas e domínio Cerrado brasileiro (SOUZA et al., 2020).

O aumento contínuo na liberação do uso de agrotóxicos a cada ano só reafirma o descaso com a saúde pública e a necessidade da indústria agrícola com a produção sem se importar com as consequências para o meio ambiente e o consumidor final.

\section{CONCLUSÕES}

Através desse estudo, conclui-se que o aumento de contaminação das águas de abastecimento por substâncias químicas pode estar relacionado com o uso indiscriminado de agrotóxicos, além de evidenciar o quanto a população do município de Campos dos Goytacazes esta suscetível a entrar em contato com essas substâncias nocivas. Dado o histórico dessas substâncias e suas elevadas concentrações na água, existe um risco maior de que a população 
venha a desenvolver problemas de saúde, a curto, médio e longo prazo. Isso se torna mais evidente uma vez que os agrotóxicos encontrados são conhecidamente maléficos à saúde humana, além de estarem acima do limite permitido pela legislação, estando ligados a danos à saúde como o câncer, defeitos congênitos e distúrbios endócrinos.

Um dos fatores que pode ter contribuído para resultados tão assustadores, é o incentivo ao uso de agrotóxicos, visando o progresso da agricultura, realizado pelo governo nos últimos anos. Com isso, inúmeros agricultores começaram a utilizar esses produtos de forma exagerada e irresponsável, objetivando apenas o aumento da sua produção e lucratividade.

A partir do que foi exposto acima, enfatiza-se um descaso com a saúde ambiental e social, com o uso exacerbado e rotineiro de agrotóxicos que precisa urgentemente ser controlado, a fim de se preservar a saúde das populações no planeta e a qualidade de vida humana, animal e vegetal dentro dos mais diversos ambientes naturais, que vem a cada ano se tornando mais preocupante.

\section{REFERÊNCIAS}

AMATO, C. D.; TORRES, J. P. M.; MALM, O. DDT (Dicloro Difenil Tricloroetano): Toxicidade e Contaminação Ambiental - Uma Revisão. Revista Química Nova, Instituto de Biofísica Carlos Chagas Filho, UFRJ, v. 25, n. 6, p. 955-1002, 2002.

ARANHA, A.; ROCHA, L. "Coquetel” com 27 agrotóxicos foi achado na água de 1 em cada 4 municípios, 2019.

BRASIL. Manual de vigilância da saúde de populações expostas a agrotóxicos. Ministério da Saúde Secretaria de Vigilância Sanitária Departamento Técnico-Normativo: Divisão de Meio Ambiente a Ecologia Humana, 1996.

CHAIM, A. O passado e o presente da tecnologia de aplicação de agrotóxicos. Tecnologia de aplicação de agrotóxicos: fatores que afetam a eficiência e o impacto ambiental, v. 8, p. 290-293, 1999.

ECOBICHÓN, D. J. Pesticide use in developing countries. Toxicology, v. 160, p. 27-33, 2001.

GEBLER, L.; SPADOTTO, C. A. Comportamento ambiental de herbicidas. In: VARGAS, L.; ROMAN, E S. (ed.). Manual de manejo e controle de plantas daninhas Bento Gonçalves: Embrapa Uva e Vinho, 2004, 57-87 p.

LANGENBACH, T. Na lógica do controle de Agrotóxicos. Revista Ciência Hoje, 1999, 62$64 \mathrm{p}$.

LOPES, C. V. A.; ALBUQUERQUE, G. S. C. DE. Agrotóxicos e seus impactos na saúde humana e ambiental: uma revisão sistemática. Saúde em Debate, v. 42, n. 117, p. 518-534, jun. 2018.

Persp. Online: biol. \& saúde, Campos dos Goytacazes, 37 (11) 19-35, 2021

seer.perspectivasonline.com.br 


\section{MORAES, R. F. de. AGROTÓXICOS NO BRASIL: PADRÕES DE USO, POLÍTICA DA REGULAÇÃO E PREVENÇÃO DA CAPTURA REGULATÓRIA, 2019, 84 p.}

MOREIRA, M. Número de agrotóxicos liberados no Brasil em 2019 é o maior dos últimos 14 anos. Folha de São Paulo, São Paulo, 28/12/2019. Disponível em: < Número de agrotóxicos liberados no Brasil em 2019 é o maior dos últimos 14 anos - 28/12/2019 - Ambiente - Folha (uol.com.br)>. Acesso em: 21 de out. 2020.

NASCIMENTO, B. L. M.; GOMES, D. R. C. S.; COSTA, G. P.; ARAÚJO, S. S.; SANTOS, L. C. A.; OLIVEIRA, J. D. Comportamento e avaliação de metais potencialmente tóxicos $(\mathrm{Cu}$ (II), $\mathrm{Cr}$ (III), $\mathrm{Pb}$ (II) e Fe(III)) em águas superficiais dos Riachos Capivara e Bacuri ImperatrizMA, Brasil. Engenharia Sanitária e Ambiental, v. 20, n. 3, p. 369-378, 2015.

NETO, M. L. F.; SARCINELLI, P. N. Agrotóxicos em água para consumo humano: uma abordagem de avaliação de risco e contribuição o processo de atualização da legislação brasileira. Engenharia Sanitária e Ambiental, v. 14, n. 1, p. 69-78, 2009.

NEVES, P. D. M., BELLINI, M. Intoxicações por agrotóxicos na mesorregião norte central paranaense, Brasil - 2002 a 2011. Revista Ciência e Saúde Coletiva, v. 18, n.11, p. 31473156, 2013.

NOGUEIRA, J. M., \& BRANCO, M. C. ANÁLISE SÓCIO-ECONÔMICO-AMBIENTAL E SUA INTERAÇÃO COM A POLÍTICA PÚBLICA: O EXEMPLO DOS AGROTÓXICOS. Biológicas \& Saúde, 1(3), 2011.

OLIVEIRA, C. F. R., \& MACEDO, M. L. R. Emprego de inibidores de protease vegetais como ferramenta biotecnológica alternativa no controle de pragas. Biológicas \& Saúde, 1(1), 2011.

ORGANIZAÇÃO PAN-AMERICANA DA SAÚDE (OPAS). Manual de vigilância da saúde de populações expostas a agrotóxicos. Brasília: Ministério da Saúde; p. 69, 1996.

PEDLOWSKI, M. A.; AQUINO, S. L.; CANELA, M. C.; I. L. A. DA SILVA, I. L. A. Um Estudo sobre a Utilização de Agrotóxicos e os Riscos de Contaminação num Assentamento de Reforma Agrária no Norte Fluminense. Journal of the Brazilian Society of Ecotoxicology, v. 1, n. 2, p. 185-190, 2006.

PERES, F.; MOREIRA, J. C. E veneno ou é remédio? agrotóxicos, saúde e ambiente. Rio de Janeiro, RJ: Editora Fiocruz, 2003.

PERES, F.; ROZEMBERG, B.; ALVES, S. R.; MOREIRA, J. C.; OLIVEIRA-SILVA, J. J. Comunicação relacionada ao uso de agrotóxicos em região agrícola do Estado do Rio de Janeiro. Revista de Saúde Pública, v. 35, n. 6, p. 564-570, 2001.

RIGOTTO, R. M.; VASCONCELOS, D. P.; ROCHA, M. M. Uso de agrotóxicos no Brasil e

Persp. Online: biol. \& saúde, Campos dos Goytacazes, 37 (11) 19-35, 2021

seer.perspectivasonline.com.br 


\section{PERSPECTIVASCnline GIÊNIAS BIOLÓGIIAS EDA SAÚdE}

problemas para a saúde pública. Cadernos de Saúde Pública, v. 30, n.7, p. 1-3, 2014.

SILVA, J. M.; SANTOS, J. R. Toxicologia de agrotóxicos em ambientes aquáticos. Departamento de Ciências Florestais, UFRPE, 2007.

SILVA, M. R.; CAMPOS, A. C. E.; BOHM, F. Z. Agrotóxicos e seus impactos sobre ecossistemas aquáticos continentais. SaBios-Revista de Saúde e Biologia, v. 8, n. 2, p.46-58, 2013.

SOUZA, M. M. O de; GURGEL, A. M.; FERNANDES, G. B.; MELGAREJO, L.; BITTENCOURT, N. A.; FRIEDRICH, K. AGROTÓXICOS E TRANSGÊNICOS: Retrocessos socioambientais e avanços conservadores no governo Bolsonaro. Revista da ANPEGE. v. 16. n. 29, p. 319 - 352, 2020.

SPADOTTO, C. A. Abordagem Interdisciplinar na avaliação ambiental de agrotóxicos. Embrapa Meio Ambiente, São Paulo: Jaguariúna, 2006.

STEFFEN, G. P. K.; STEFFEN, R. B.; ANTONIOLLI, Z. I. Contaminação do solo e da água pelo uso de agrotóxicos. Revista do Departamento de Química e Física, Universidade Federal de Santa Maria, Santa Maria, v.15, n. 1, 2011.

SUDRÉ, L. Liberação de agrotóxicos no governo Bolsonaro é a maior dos últimos 14 anos. Brasil de Fato, São Paulo, 27/11/2019. Disponível em: <Liberação de agrotóxicos no governo Bolsonaro é a maior dos | Geral (brasildefato.com.br)>. Acesso em: 21 de out. 2020.

TEIXEIRA, D. E.; DUARTE, V. S.; NAPOLITANO, H. B.; \& SILVA, S. D. A expansão da fronteira agrícola e a utilização do Dicloro-Difenil-Tricloroetano (DDT) em Goiás (19401980). Revista Inclusiones, v. 6, n. 4, 2019.

VEIGA, M. M.; SILVA, D. M.; VEIGA, L. B. E.; MARIA, M. V. C. Análise da contaminação dos sistemas hídricos por agrotóxicos numa pequena comunidade rural do Sudeste do Brasil. Cadernos de Saúde Pública, Rio de Janeiro, v. 22, n. 11, p. 2391-2399, 2006.

WORLD HEALTH ORGANIZATION; UNITED NATIONS ENVIRONMENT PROGRAMME (EDS). Public health impact of pesticides used in agriculture. Geneva: World Health Organization, 1990.

WORLD RESOURCES INSTITUTE (WRI); OXFORD UNIVERSITY PRESS. A Guide to the Global Environment. Washington, 1998. 